\title{
Theoretical Basis for the Dubinin-Radushkevitch (D-R) Adsorption Isotherm Equation
}

\author{
NICK D. HUTSON AND RALPH T. YANG \\ Department of Chemical Engineering, University of Michigan, Ann Arbor, MI 48109
}

Received March 18, 1996; Revised June 26, 1996; Accepted July 10, 1996

\begin{abstract}
The Dubinin-Radushkevitch (D-R) equation, which was originally proposed as an empirical adaptation of the Polanyi adsorption potential theory, has been the fundamental equation to quantitatively describe the adsorption of gases and vapors by microporous sorbents. The equation, based on the postulate that the mechanism for adsorption in micropores is that of pore-filling rather than layer-by-layer surface coverage, generally applies well to adsorption systems involving only van der Waals forces and is especially useful to describe adsorption on activated carbon. The ability of the D-R equation to describe gas adsorption on porous materials has inspired many to undertake studies, both experimental and theoretical, to explain the source of the success of the D-R equation in terms of molecular properties at the gas-solid interface. In many cases, these studies have led to extensions or modifications of the original D-R equation. Many of these attempts and the resulting extensions are reviewed and discussed here. Recently, an isotherm equation was derived for adsorption of gases and vapors on microporous solids from statistical mechanical principles. It was shown that the D-R equation is an approximated form of this potential theory isotherm. This development is also reviewed and discussed.
\end{abstract}

Keywords: Dubinin-Radushkevitch equation, Dubinin-Astakhov equation, adsorption, micropore adsorption

\section{Introduction}

\section{Background}

Most adsorbents used in modern technology, such as activated carbons, synthetic and natural zeolites, silica and other inorganic gels, etc., are microporous in nature (i.e., they contain pores with equivalent radii less than 6-7 A). And while these sorbents may contain larger pore varieties (e.g., mesopores and macropores), it is the micropores that determine the adsorptive nature of the material. Despite intensive study, much of it by Dubinin and coworkers, there are still fundamental questions in the science of adsorption of gases and vapors by microporous solids which remain unanswered (Rudzinski and Everett, 1992). Nonetheless, for nearly a half-century, the Dubinin-Radushkevitch equation has been used to effectively describe the adsorption of vapors and gases by microporous solids.

\section{Development of the D-R Equation}

The development of this equation was prompted by the failure to extend the theories governing adsorption on non-porous solids to that on real, porous solids such as active carbons, synthetic zeolites, and dehydrated inorganic gels (Dubinin, 1966, 1975). This lack of success led Dubinin and co-workers to focus their investigations on the problem of physical adsorption on porous materials and resulted in the Dubinin-Polanyi theory of micropore filling (also known as the theory of volume filling of micropores (TVFM)). This theory is based on the postulate that the mechanism for adsorption in micropores is that of pore filling rather than a layer-by-layer formation of a film on the walls of the pores.

The $D-R$ equation is an adaptation of the earlier Polanyi potential theory of adsorption (Polanyi, 1914, 1932; Goldmann and Polanyi, 1928; Gregg and Sing, 
1982). An essential parameter of this theory is the quantity $\mathrm{A}$, defined by

$$
A=R T \ln \left(\frac{P^{0}}{P}\right)
$$

where $P$ is the equilibrium pressure at temperature $T$, $P^{0}$ is the saturated vapor pressure, and $A$ is referred to, by Polanyi, as the adsorption potential. Although, the development of this theory requires an assumption of layer-by-layer coverage of the pore surfaces with the formation of a condensed adsorption film on the walls of the micropores and thus contradicts Dubinin's fundamental postulate, the basic principle of the theory concerning the temperature invariance of the parameter $A$ was shown to be true for microporous adsorbents but not, to a satisfactory degree, for large-pore and nonporous adsorbents (Dubinin, 1975).

Dubinin and coworkers adopted a thermodynamic interpretation of the Polanyi adsorption potential, referring to it as the differential molar work of adsorption and expressing it as the negative differential free energy of adsorption $(\Delta G)$ :

$$
A=-\Delta G=R T \ln \left(\frac{P^{0}}{P}\right)
$$

Since Dubinin considered microporous adsorption to be a process of volume filling, a second parameter, the degree of filling of the micropores $\theta$ was introduced and defined by

$$
\theta=\frac{W}{W_{0}}
$$

where $W_{0}$ is the total volume of the micropore system and $W$ is the volume that has been filled when the relative pressure is $P / P^{0}$. A fundamental postulate in the development of this theory is that $\theta$ is a function of $A$ such that

$$
\theta=f(A / E)
$$

or

$$
\theta=f\left(\frac{A}{E_{0} \beta}\right)
$$

where $A$ is the differential molar work of adsorption, $E$ is the characteristic energy of adsorption, $E_{0}$ is the characteristic energy of adsorption for a reference vapor (usually benzene), and $\beta$ is termed the similarity constant (where $\beta=1$ for the reference vapor). The reason for grouping $A / E$ is simply to express it as a dimensionless unit. On the assumption that the pore size distribution is Gaussian, Dubinin and Radushkevitch (1947) arrived at the following expression:

$$
\theta=\exp \left[-\left(\frac{A}{E_{0} \beta}\right)^{2}\right]
$$

which is the commonly used form of the D-R equation. Combining Eqs. (2), (3) and (6) results in the following expression:

$$
W=W_{0} \exp \left[-\left(\frac{R T \ln \frac{P^{\prime \prime}}{P}}{E_{0} \beta}\right)^{2}\right]
$$

or

$$
W=W_{0} \exp \left[-B\left(\frac{T}{\beta} \log \frac{P^{0}}{P}\right)^{2}\right]
$$

where $E_{0}$ and $B$ are related by the following expression:

$$
E_{0}=0.001915 \sqrt{(1 / B)}
$$

\section{Uses and Limitations of the D-R Equation}

The D-R and D-A equations have been effectively used to describe adsorption by microporous solids (Huber et al., 1978). The D-R and D-A equations seem to be particularly useful in describing adsorption by microporous carbonaceous sorbents or activated carbons (Dubinin and Stoeckli, 1980). In general, the TVFM seems to be most applicable for microporous solids with practically homogeneous or uniform micropore structures (Dubinin, 1981; Dubinin and Kadlec, 1987; Jaroniec et al., 1989) and least applicable for microporous adsorbents with a wide micropore distribution (Dubinin, 1981).

The principal deficiency of the D-R equation is its behavior in the limit of zero loading (i.e., as $\theta \rightarrow 0$ ) Hacskaylo and LeVan (1985) and Dubinin (1975) has pointed out that the validity of the TVFM (and thus the D-R equation) is limited to degrees of micropore filling higher than 0.15 . The $D-R$ isotherm does not reduce to Henry's law at low pressures, which is a necessity for a thermodynamically consistent isotherm (Chen and Yang, 1994). In the low loading region, a thermodynamically consistent adsorption isotherm should give a finite Henry's law slope. The D-R equation, however 
gives a Henry's law slope equal to zero in this region (Kapoor et al., 1989).

\section{Theoretical Basis of the D-R Equation}

The studies of Hobson (1961) revealed the ability of the D-R equation to describe gas adsorption on nonporous, macroporous, and mesoporous adsorbents. This work inspired many others to undertake studies, both experimental and theoretical, in an effort to explain the source of the success of the D-R equation (Jaroniec and Madey, 1988). In many cases, these studies have led to extensions or modifications of the original $D-R$ equations. $A$ few of these are summarized below.

\section{Dubinin-Astakhov (D-A) Equation}

Dubinin and Astakhov (1971) further expanded Eq. (4) with the addition of an additional parameter $n$, such that

$$
\theta=f(A / E, n)
$$

The parameter $n$ was included in this development since most distribution functions in the normalized form are characterized by two parameters (Dubinin, 1975). Assuming the temperature invariance of Eq. (10) and the Weibull distribution known in mathematical statistics, Dubinin and Astakhov (1971) obtained an equation of adsorption in an analytical form. Weibull's distribution function $F(A)$ in normalized form is expressed as:

$$
F(A)=1-\exp \left[-\left(\frac{A}{E}\right)^{n}\right]
$$

From Eqs. (11) and (2), one can see that at $A=0$ (i.e., $\left.P / P^{0}=1\right), F(A)=0$. On the other hand, as $P / P^{0}$ decreases to low equilibrium relative pressures, $A$ reaches very high values, and $F(A)$ tends to zero. Therefore, $F(A)$ can be assumed to express the portion of the unfilled adsorption volume of the micropores (i.e., $1-\theta$ ). So, then

$$
F(A)=1-\theta=1-\exp \left[-\left(\frac{A}{E}\right)^{n}\right]
$$

which simplifies to the Dubinin-Astakhov equation:

$$
\theta=\exp \left[-\left(\frac{A}{E}\right)^{n}\right]
$$

or

$$
\theta=\exp \left[-\left(\frac{A}{E_{0} \beta}\right)^{n}\right]
$$

The D-A equation is simply a more generalized version of the D-R equation (where the parameter $n=2$ ). Experimental evidence reveals that $n \approx 2$ for activated carbonaceous sorbents (Dubinin, 1975) and approximately 4-6 for zeolites. The term $n$ has been referred to as a "heterogeneity factor" (Rudzinski and Everett, 1992); and, it has been suggested that it is related in some way to the heterogeneity (with respect to the pore distribution) of the surface.

\section{Extensions by Cerofolini and Stoeckli}

Cerofolini $(1972,1974)$ postulated a relationship between the heterogeneity of the sorbent surface and the adsorptive properties of that surface. He assumed that heterogeneous surfaces are comprised of numerous homogeneous "patches" and that the overall isotherm $\left(\theta_{t}\right)$ for the adsorptive system is related to a local isotherm $\left(\theta_{l}\right)$ for the patches and a distribution of adsorptive energies $N(\epsilon)$ by the following:

$$
\theta_{t}(T ; P)=\int_{\epsilon_{0}}^{\infty} \theta_{l}(T ; P ; \epsilon) \aleph(\epsilon) d \epsilon
$$

where $\epsilon_{0}$ is $>0$ and corresponds to the local minimum of the adsorption potential resulting from intermolecular forces.

Cerofolini (1974) showed that Eq. (15) reduces to

$$
\theta_{t}(T ; P)=\int_{\epsilon_{0}}^{\infty} \aleph_{c}(\epsilon) d \epsilon
$$

or

$$
\aleph_{c}(\epsilon)=-\partial \theta_{\imath} / \partial \epsilon
$$

using a technique called the condensation approximation (CA). This formed the foundation for a proposal by Stoeckli (1981) for the theoretical basis of the D-A 
equation (and thus the D-R equation). Stoeckli proposed that the D-R and D-A equations, which were proposed as semi-empirical relationships with some thermodynamic considerations, could be based on a theoretical model involving adsorption energies and their distribution (Stoeckli, 1990). In the development of this theory, Stoeckli postulated that if the local isotherm $\left(\theta_{l}\right)$ is the Langmuir type, an overall isotherm $\left(\theta_{t}\right)$ of the D-R type

$$
\theta_{t}=\exp \left(-B\left(R T \ln \frac{p_{m}}{p}\right)^{2}\right)
$$

corresponds to the energy distribution

$$
\aleph_{c}(\epsilon)=2 B\left(\epsilon-\epsilon_{0}\right) \exp \left(-B\left(\epsilon-\epsilon_{0}\right)^{2}\right)
$$

where, by the condensation approximation,

$$
\epsilon-\epsilon_{0}=R T \ln \left(\frac{p_{m}}{p}\right)
$$

So, in a generalization of this technique, using the following assumptions:

(1) the overall isotherm is the D-A equation (Eq. (14)),

(2) the local isotherm in the micropore is of the Langmuir type,

(3) $P_{m}=P^{0}$,

(4) for carbons $\epsilon_{0}$ is close to the adsorption energy on the open surface,

the condensation approximation method leads to the following approximate energy distribution associated with the D-A equation:

$\aleph_{c}(\epsilon)=n \frac{\left(\epsilon-\epsilon_{0}\right)^{n-1}}{\left(\beta E_{0}\right)^{n}} \exp \left(-\left(\frac{\epsilon-\epsilon_{0}}{\beta E_{0}}\right)^{n}\right)$.

In this approach, the exponent $n$ reflects the width of the energy distribution and is related to the pore size distribution (Stoeckli, 1990).

\section{Dubinin-Radushkevitch-Stoeckli (D-R-S) Equation}

Temperature invariance was the basic premise in formulating the D-R equation. However, it was noted by Stoeckli (1977), Dubinin and coworkers (1980) that the characteristic energy of adsorption $\left(E_{0}\right)$ decreases with increasing differential work of adsorption $(A)$. This observation led Stoeckli (1977) to postulate that the
D-R equation applies only to structurally homogeneous systems, i.e., micropores with the same dimension. As a result, Stoeckli (1977) and Huber et al. (1978) attempted to generalize the D-R equation to adsorbents with inhomogeneous microporous structures. For this purpose they assumed a normal Gaussian distribution of micropores $W_{0}$ with respect to the parameter $B$ of the D-R Eq. (8):

$$
\frac{d W_{0}}{d B}=f(B)=\frac{W_{0}^{0}}{\Delta \sqrt{2 \pi}} \exp \left[-\frac{B_{0}-B}{2 \Delta^{2}}\right]
$$

where $W_{0}^{0}$ is the total micropore volume, $B_{0}$ is the value of the parameter $B$ for the minimum of the distribution curve, and $\Delta$ is the dispersion (or variance). One can write the following equation for an adsorbent with an inhomogeneous microporous structure in accordance with Eqs. (8) and (22):

$$
W=\int_{0}^{\infty} f(B) \exp [-B y] d B .
$$

where

$$
y=\left[\frac{T}{\beta} \log \frac{P^{0}}{P}\right]^{2}
$$

Integrating (23) yields the adsorption equation:

$$
W=W_{0}^{0}\left[\exp \left(-B_{0} y\right)\right] \exp \left[0.5 y^{2} \Delta^{2}\right] 0.5[1-\operatorname{erf}(z)]
$$

where

$$
z=\left(y-\frac{B_{0}}{\Delta^{2}}\right) \frac{\Delta}{\sqrt{2}} .
$$

Equation (25) is referred to as the DubininRadushkevitch-Stoeckli (D-R-S) equation. Equation (23) can be rewritten as follows:

$$
\theta=\int_{0}^{\infty} \exp \left[-\left(\frac{A}{E}\right)^{2}\right] g(E) d E
$$

where $g(E)$ is the energy distribution function of the micropores and $E$ is the local characteristic energy and empirically, $E$ is related to pore dimension by (Dubinin and Stoeckli, 1980)

$$
E=X / x
$$

where $x$ is the half-width of the slit pore and $X$ is an empirical constant. 


\section{Extensions by Jaroniec et al.}

As mentioned, Dubinin and Stoeckli assumed a Gaussian micropore-size distribution in the development of the D-R-S equation. Jaroniec and coworkers (1988a, 1989, 1991) proposed a gamma-type distribution of the following form:

$$
f(B)=\frac{q^{n+1}}{\Gamma(n+1)} B^{n} \exp (-q B)
$$

where $q>0, n>-1$ and $\Gamma$ is the gamma function. The distribution $f(B)$ is defined on the domain $[0, \infty)$ and generates the following micropore-size distribution (Jaroniec et al., 1989):

$$
G(x)=\frac{\left(q_{c}\right)^{n+1}}{\Gamma(n+1)} x^{2 n+1} \exp \left(-q_{c} x^{2}\right)
$$

Using the relationship,

$$
\theta_{t}=\int_{0}^{\infty} \exp \left[-B\left(\frac{A}{\beta}\right)^{2}\right] f(B) d B
$$

the distribution $f(B)$ in Eq. (31) generates the following simple equation:

$$
\theta_{t}=\left[\frac{q}{q+(A / \beta)^{2}}\right]^{n+1}
$$

This equation is referred to as the Jaroniec-Choma (or $\mathrm{J}-\mathrm{C}$ ) equation.

\section{Potential Theory Isotherm}

Chen and Yang (1994) have derived an isotherm equation from statistical mechanical principles which, for appreciable degrees of pore filling, e.g., $\theta>0.1$ reduces to the D-A and D-R equations. For $\theta \rightarrow 0$, the isotherm equation reduces to Henry's law (which is not true and is the primary flaw of the D-R equation). In the derivation of this equation, Chen and Yang considered a system of $N$ fluid molecules exposed to a force field within a given pore size and geometry. Each molecule in this force field has a potential energy of $\Phi\left(r_{k}\right)$, where $r_{k}$ is the position coordinate of molecule $k$ in the system. The partition function $Z_{N}^{s}$ of the canonical ensemble of the system can be expressed as (Steele, 1974):

$$
\begin{aligned}
Z_{N}^{*}= & \frac{q_{r} q_{v}}{N ! \Lambda^{\lambda N}} \int_{V^{s}} d r^{N} \\
& \times \exp \left(-\gamma \sum_{i<j}^{N} U\left(\dot{r}_{i j}\right)-\gamma \sum_{k}^{N} \Phi\left(r_{k}\right)\right)
\end{aligned}
$$

where $q_{r}$ and $q_{v}$ are the rotational and vibrational partition functions of the $N$ fluid molecules and are separated from the translational partition function; the superscript $s$ denotes the adsorbed phase. $\lambda$ is a constant indicating the dimensions of the space (i.e., $\lambda=3$ for three dimensional space and $\lambda=2$ for two dimensional space). For adsorption in micropores, however, $\lambda$ may lie between 2 and $3 . U\left(r_{i j}\right)$ is the potential energy between two molecules " $i$ " and " $j$ " at distance $r_{i j}$, and $\Phi\left(r_{k}\right)$ is the potential energy of molecule " $k$ " at $r_{k}$, which arises from the force field in the pore. $V^{s}$ is the volume of the system, $d r^{N}=d r_{1} d r_{2} \cdots d r_{N}$ for all positions, $\gamma=1 /(k T)$ and

$$
\Lambda=h /(2 \pi m k T)^{1 / 2}
$$

is the de Broglie thermal wavelength, $h$ is Planck's constant, $m$ is the molecular weight, $k$ is Boltzmann's constant, and $T$ is the absolute temperature.

Although the potential energy is a function of position in the pore, Chen and Yang (1994) assumed that the force field may be approximated by a mean value, $\Phi$, for all adsorbate molecules in the pore. By using a two-dimensional equation of state to represent the adsorbed phase and equating the chemical potentials of the adsorbed and gas phases, the resulting adsorption isotherm equation was then derived as follows:

$$
\begin{aligned}
& \ln \left(\frac{\rho^{s}}{\rho^{8} \Lambda}\right)-\frac{7}{8} \ln (1-\eta)+\frac{2 \eta}{(1-\eta)}+\frac{9}{8} \frac{\eta}{(1-\eta)^{2}} \\
& \quad-\alpha(T) \eta+\frac{\Phi}{k T}=0
\end{aligned}
$$

where $\rho^{s}$ and $\rho^{g}$ are number densities of the adsorbed and fluid phases, respectively. The quantity $\eta$ represents the fraction of the surface that is covered (the packing fraction at the surface), $\Lambda$ is the de Broglie thermal wavelength, $k$ is the Boltzmann constant, and $\alpha(T)$ is a function of $T$ only.

In the derivation of the $\mathrm{D}-\mathrm{R}$ equation, the isotherm at pressure $P^{0}$,

$$
\begin{aligned}
& \ln \left(\frac{\rho_{0}^{s}}{\rho_{0}^{g} \Lambda}\right)-\frac{7}{8} \ln \left(1-\eta_{0}\right)+\frac{2 \eta_{0}}{\left(1-\eta_{0}\right)}+\frac{9}{8} \frac{\eta_{0}}{\left(1-\eta_{0}\right)^{2}} \\
& -\alpha(T) \eta_{0}+\frac{\Phi}{k T}=0
\end{aligned}
$$

' was subtracted from that at pressure $P$ (as given in 
Eq. (35)). The result expression is as follows:

$$
\ln \left(\frac{\rho^{*}}{\rho_{0}^{*}}\right)+F(\eta)-F\left(\eta_{0}\right)=\ln \left(\frac{P}{P_{0}}\right)
$$

where

$$
\begin{aligned}
F(\eta)= & -\frac{7}{8} \ln (1-\eta)+\frac{2 \eta}{(1-\eta)} \\
& +\frac{9}{8} \frac{\eta}{(1-\eta)^{2}}-\alpha(T) \eta
\end{aligned}
$$

and

$$
\frac{\rho^{g}}{\rho_{0}^{g}}=\frac{P}{P_{0}} .
$$

Since, in the D-R and D-A equations, $\theta$ is the fractional pore filling relative to the saturation vapor pressure, the following is true

$$
\frac{\rho^{s}}{\rho_{0}^{s}}=\frac{\eta}{\eta_{0}}=\theta
$$

A purely mathematical approximation, that

$$
F(\eta)-F\left(\eta_{0}\right) \cong-K F\left(\eta_{0}\right)(-\ln \theta)^{1 / n}
$$

where $K$ and $n$ are constants resulting from the approximation and have no physical meaning, is made and Eq. (37) may be mathematically manipulated to:

$$
\begin{aligned}
\ln \theta & =-\left[\frac{k T}{K \Phi} \ln \left(\frac{P}{P^{0}}\right)\right]^{n} \\
& =-\left[\frac{R T}{K N_{0} \Phi} \ln \left(\frac{P}{P^{0}}\right)\right]^{n}
\end{aligned}
$$

where $N_{0}$ is the Avogadro number. Chen and Yang (1994) showed that the mathematical approximation of Eq. (41) is good for $\theta=0.2-1.0$, but breaks down when $\theta<0.1$. This is shown in Fig. 1 where the approximations of Eq. (41) and the further case where $\ln \theta$ is neglected are shown for the representative case of $\eta_{0}=0.65$. Dubinin (1975) and others have pointed out this problem for the D-R and D-A equations.

Equation (42) is the form of the Dubinin-Astakhov equation (or the D-R equation when $n=2$ ) where

$$
K N_{0} \Phi=E_{0} \beta
$$

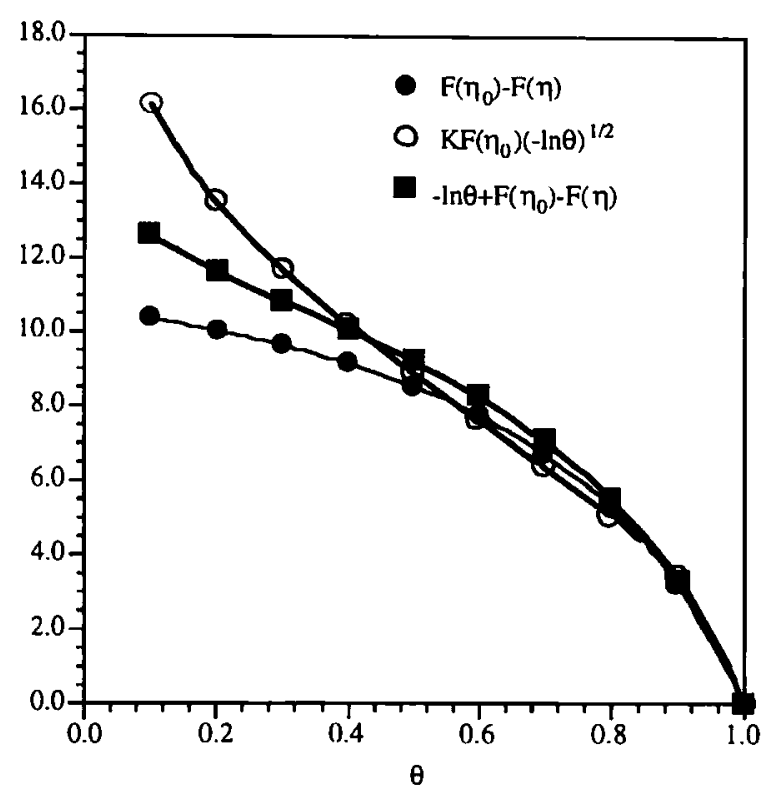

Figure 1. Goodness of approximation for Eq. (41), for the representative case of $\eta_{0}=0.65$.

Equation (43) shows that the characteristic energy of adsorption $\left(E_{0} \beta\right)$ in the D-R (and D-A) equation is directly related to the mean potential $(\Phi)$ in the pore. Since the dependence of $\Phi$ on pore size can be established it is possible to investigate the dependence of $E_{0} \beta$ on pore size.

\section{Nomenclature}

$A$ adsorption potential; differential molar work of adsorption; Helmholtz free energy

$B$ parameter in D-R, D-A equations, related to $E_{0}$

$B_{0} \quad$ value of the parameter $B$ for the minimum of the micropore distribution curve in the D-R-S equation

$E$ characteristic energy of adsorption; energy

$E_{0}$ characteristic energy of adsorption for standard vapor

$g(E)$ energy distribution function

$G(x)$ micropore size distribution

$h$ Planck's constant

$k$ Boltzmann's constant

$K$ constant from approximation, Eq. (41)

$m$ molecular weight

$n$ parameter in D-A equation

$N$ number of molecules in the system

$N_{0} \quad$ Avogadro's number 
$P$ pressure

$P_{m} \quad$ condensation pressure

$p^{0} \quad$ saturated vapor pressure

$q$ parameter of the Gamma micropore distribution

$q_{c}$ parameter of the Gamma micropore distribution for a reference vapor

$q_{r}$ rotational partition function

$q_{v}$ vibrational partition function

$R$ gas constant

$r_{k}$ position coordinate of molecule " $k$ "

$T$ temperature

$U\left(r_{i j}\right)$ potential energy between molecules $i$ and $j$

$V^{s} \quad$ surface area

$W$ volume of micropores filled at relative pressure $P / P^{0}$

$W_{0}$ total volume of micropore system

$x$ half-width of slit pore

$X$ empirical constant relating characteristic energy to pore size

$Z_{N}^{*} \quad$ partition function of the canonical ensemble

\section{Greek etc. Symbols}

$\begin{aligned} \alpha(T) & \text { a function of temperature in Eq. (35) } \\ \beta & \text { similarity coefficient } \\ \Delta & \text { dispersion (or variance) } \\ \Delta G_{\text {ads }} & \text { differential free energy of adsorption } \\ \epsilon & \text { energy } \\ \epsilon_{0} & \text { minimum adsorption energy } \\ \eta & \text { packing fraction of the surface } \\ \eta_{0} & \text { packing fraction of the surface at } P=P^{0} \\ \gamma & 1 / k T \\ \Gamma & \text { gamma function } \\ \lambda & \text { number of dimension } \\ \Lambda & \text { de Broglie thermal wavelength } \\ \Phi & \text { potential energy function } \\ \rho^{s} & \text { number density of the solid phase } \\ \rho^{g} & \text { number density of the gas phase } \\ \theta & \left.\text { fractional coverage (=W/W } W_{0}\right) \\ \theta_{t} & \text { overall isotherm for microporous system } \\ \theta_{l} & \text { local isotherm for microporous system } \\ \kappa(\epsilon) & \text { energy distribution }\end{aligned}$

\section{Acknowledgments}

This work was supported by a grant from the National Science Foundation (NSF Grant CTS-9520328) and partially by the BOC Group.

\section{References}

Cerofolini, G.F., J. of Low Temp. Physics, 6, 473 (1972).

Cerofolini, G.F., Thin Solid Films, 23, 129 (1974).

Cerofolini, G.F., J. of Colloid and Interface Sci, 86, 204 (1982).

Chen, S.G. and R.T. Yang, Langmuir, 10, 4244 (1994).

Dubinin, M.M., Chem. and Phys. of Carbon, P.L. Walker, Jr. (Ed.), Mercel Dekker, New York, 1966.

Dubinin, M.M., Progress in Surface and Membrane Science, D.A. Cadenhead et al. (Eds.), Academic Press, New York, 1975.

Dubinin, M.M., Carbon, 23, 373 (1985).

Dubinin, M.M. and O. Kadlec, Carbon, 24, 321 (1987).

Dubinin, M.M. and V.A. Astakhov, Izv, Akad. Nauk SSR, Ser. Khim., p. 5,1971 .

Dubinin, M.M. and L.V. Radushkevitch, Proc. Acad. Sci. USSR., Vol. 55, p. 331, 1947.

Dubinin, M.M. and H.F. Stoeckli, J. of Colloid and Interface Sci., 75, 34 (1980).

Goldmann, F. and M.M. Polanyi, Z. Phys. Chem., Abt. A, 132, 321 (1928).

Gregg, S.J, and K.S.W. Sing, Adsorption, Surface Area and Porosity, 2nd Ed., Academic Press, London, 1982.

Hacskaylo, J.J. and M.D. LeVan, Langmuir, 1, 97 (1985).

Hobson, J.P., J. of Phys. Chem., 34, 1850 (1961).

Huber, H., H.F. Stoeckli, and J.Ph. Houriet, J. of Colloid and Interface Sci., 67, 195 (1978).

Jaroniec, M. and J. Choma, Carbon, 26, 747 (1988a).

Jaroniec, M. and R. Madley, Physical Adsorption on Heterogeneous Solids, Elsevier, Amsterdam, 1988b.

Jaroniec, M., R. Madey, J. Choma, B. McEnaney, and T.J. Mays, Carbon, 27, 77 (1989).

Jaroniec, M., J. Choma, and X. Lu, Chem. Eng. Sci., 46, 3299 (1991).

Kapoor, A., J.A. Ritter, and R.T. Yang, Langmuir, 5, 1118 (1989).

Polanyi, M., Verb. Deutsch. Physik. Ges., 16, 1012 (1914).

Polanyi, M., Trans. Faraday Soc., 28, 316 (1932).

Rudzinski, W. and D.H. Everett, Adsorption of Gases on Heterogeneous Surfaces, Academic Press, London, 1992.

Steele, W.A., The Interaction of Gases with Solid Surfaces, Pergamon Press, Oxford, 1974.

Stoeckli, H.F., J. of Colloid and Interface Sci., 59, 184 (1977).

Stoeckli, H.F., Carbon, 19, 325 (1981). 